\title{
CRIRES: Commissioning and First Science Results
}

\author{
Hans Ulrich Käuff ${ }^{a}$, Paola Amico ${ }^{b}$, Pascal Ballester ${ }^{a}$, Eduardo A. Bendek Selman ${ }^{b}$, \\ Paul Bristow ${ }^{a}$, Mark Casali ${ }^{a}$, Bernard Delabre ${ }^{a}$, Danuta Dobrzycka ${ }^{a}$, Reinhold J. Dorn ${ }^{a}$, \\ Raul Esteves ${ }^{c}$, Gert Finger ${ }^{a}$, Gordon Gillet ${ }^{b}$, Domingo Gojak ${ }^{a}$, Michael Hilker ${ }^{a}$, \\ Paul Jolley ${ }^{a}$, Yves Jung ${ }^{a}$, Florian Kerber ${ }^{a}$, Barbara Klein $^{a}$, Jean-Louis Lizon ${ }^{a}$, \\ Jerome Paufique $^{a}$, Jean-Francois Pirard ${ }^{a}$, Eszter Pozna ${ }^{a}$, Hugues Sana $^{b}$, Lilian Sanzana $^{b}$, \\ Ricardo Schmutzer ${ }^{b}$, Andreas Seifahrt ${ }^{d}$, Ralf Siebenmorgen ${ }^{a}$, Alain Smette $^{b}$, \\ Jörg Stegmeier ${ }^{a}$, Lowell E. Tacconi-Garman ${ }^{a}$, Stefan Uttenthaler ${ }^{e}$, Elena Valenti ${ }^{b}$, \\ Ueli Weilenmann ${ }^{b}$, and Burkhard Wolf ${ }^{a}$ \\ ${ }^{a}$ European Southern Observatory, Karl-Schwarzschild-Strasse 2, \\ D-84758 Garching bei München, Germany \\ ${ }^{b}$ European Southern Observatory, Alonso de Cordova 3107, Santiago 19, Chile \\ ${ }^{c}$ Rua do Verdelho n40, 4470-825 Maia, Porto, Portugal \\ ${ }^{d}$ Universität Göttingen, Institut für Astrophysik, Friedrich-Hund-Platz 1, \\ 37077 Göttingen, Germany \\ ${ }^{e}$ Instituut voor Sterrenkunde, KU Leuven, Celestijnenlaan 200B, 3001 Leuven, Belgium
}

\begin{abstract}
CRIRES is a cryogenic, pre-dispersed, infrared Echelle spectrograph designed to provide a nominal resolving power $\nu / \Delta \nu$ of $10^{5}$ between 1000 and $5000 \mathrm{~nm}$ for a nominal slit width of 0.2 ". The CRIRES installation at the Nasmyth focus A of the 8-m VLT UT1 (Antu) marks the completion of the original instrumentation plan for the VLT. A curvature sensing adaptive optics system feed is used to minimize slit losses and to provide 0.2" spatial resolution along the slit. A mosaic of four Aladdin InSb-arrays packaged on custom-fabricated ceramic boards has been developed. It provides for an effective $4096 \times 512$ pixel focal plane array to maximize the free spectral range covered in each exposure. Insertion of gas cells is possible in order to measure radial velocities with high precision. Measurement of circular and linear polarization in Zeeman sensitive lines for magnetic Doppler imaging is foreseen but not yet fully implemented. A cryogenic Wollaston prism on a kinematic mount is already incorporated. The retarder devices will be located close to the Unit Telescope focal plane. Here we briefly recall the major design features of CRIRES and describe the commissioning of the instrument including a report of extensive testing and a preview of astronomical results.
\end{abstract}

Keywords: Infrared Spectroscopy, Molecular Spectroscopy Stellar Atmospheres, Interstellar Medium, Calibration

\section{INTRODUCTION}

CRIRES is the last instrument of the original instrumentation plan for ESO's VLT. Heritage, detailed design and fabrication have been reported previously. ${ }^{1}$ CRIRES opens up a parameter space in the near infrared, not generally available with facility class instruments at large telescopes. The spectrograph combines near-diffraction limited spatial resolution with a spectral resolution equivalent to a Doppler-width of $3 \mathrm{~km} / \mathrm{s}$. This allows for a great variety of investigations from the study of Quasar absorption lines to rotational-vibrational transitions in carbon-bearing molecules emanating from comets in the solar system. For the full scope of science cases see e.g. the proceedings ${ }^{2}$ of a special workshop held at ESO-Garching in November 2003. Particularly unique is a novel access to molecules, an area so far largely restricted to radio- and sub-mm astronomy. As all IR-observations, CRIRES is least affected by extinction through dust and gas and reddening corrections are, if necessary at all, small. Even though CRIRES is unique and only in regular operations since April 2007 it already is an extremely popular instrument, contributing its share to the oversubscription of the ESO-VLT.

Further author information: (Send correspondence to H.U.K.)

H.U.K.: E-mail: hukaufl@eso.org, Telephone: +49 8932006414 


\section{DESCRIPTION OF INSTRUMENT}

The optical layout is shown and described in figure 1. The CRIRES adaptive optics module, ${ }^{3} \# 6$ out of a series of six curvature sensing adaptive optics systems built for the ESO-VLT ${ }^{4}$ has shown impressive results during its commissioning. ${ }^{5}$ The system closes the loop under normal conditions for a natural guide star R-magnitude of 16-17. As the CRIRES nominal slit width (0.2") is less than the diffraction limit of an $8 \mathrm{~m}$ telescope* slit losses are usually acceptable if not negligible. The overall properties of CRIRES are described in table 1. More details are given in the user's manual (c.f. http://www.eso.org/sci/facilities/paranal/instruments/crires/). The CRIRES detector mosaic consists of four detectors which have been hybridized to special custom fabricated

Table 1. Overview of CRIRES main characteristics in a nut-shell.

\begin{tabular}{|l|l|}
\hline spectral coverage & $\begin{array}{l}\nu: 58.000-310.000 \mathrm{GHz} \\
(\lambda: 950-5200 \mathrm{~nm})\end{array}$ \\
\hline spectral resolution & $\begin{array}{l}\nu / \Delta \nu \approx 10^{5} \text { or } 3 \mathrm{~km} / \mathrm{s} \\
(2 \text { pixel Nyquist sampling })\end{array}$ \\
\hline spatial resolution along slit & $0.2^{\prime \prime}\left(2\right.$ pixel Nyquist sampling), slit-length $\approx 40^{\prime \prime}$ \\
\hline array detector mosaic & $\begin{array}{l}4 \times 1024 \mathrm{x} 512 \text { Aladdin III InSb arrays } \\
\text { instantaneous } \nu(\lambda) \text { coverage } \geq 2.0 \%\end{array}$ \\
\hline infrared slit viewer & Aladdin III, with J,H \& K-filters, scale $0.05 " /$ pix \\
\hline precision for calibration and stability & $\approx 75 m / s$ i.e. $1 / 20^{t h}$ of a pixel or 5 mas tracking error $($ goal $)$ \\
\hline dark current & measured $($ optical \& detector $) \leq 1 / 20^{t h} e^{-} / \mathrm{s}$ \\
\hline sensitivity & $\begin{array}{l}\text { read-out noise or background noise limited } \\
\text { for details see the exposure time calculator } \\
\text { http: //www. eso.org/observing/etc/ }\end{array}$ \\
\hline ghosts, stray light & $\begin{array}{l}\text { specification } \leq 10^{-3} ; \text { achieved } \leq 10^{-4} ; \text { exception, in the near IR } \\
\text { a white light ghost, however displaced form the spectrum }\end{array}$ \\
\hline
\end{tabular}

ceramics. The arrays are rotated such that always the best two of the four quadrants are illuminated by the spectrum. While the overall characteristics of this detector assembly have already been reported elsewhere, ${ }^{6}$ it should be noted that this was a rather economic approach in achieving a mega-pixel focal plane, albeit at the price, that the readout direction is for two chips perpendicular and for two parallel to the dispersion which puts an extra burden on flat-fielding and data processing.

CRIRES was designed and built by ESO in house under the supervision of and in consultation with its science team ${ }^{\dagger}$.

\section{EXPERIENCE FROM COMMISSIONING}

\subsection{Calendar of Integration and Commissioning}

CRIRES had been pre-erected and tested in Garching integrated with its AO-module. The AO-module was shipped in early 2006 to Chile and commissioned separately ${ }^{5}$ with a special camera, as CRIRES does not have sufficient spatial resolution to sample the diffraction limited PSF of an $8 \mathrm{~m}$-telescope. The cryostat was shipped in April 2006 to Chile. No transport damages worth noticing were experienced. CRIRES was then re-erected in the Paranal instrumentation laboratory and thoroughly tested, before it was transferred to its final location, the Nasmyth-A-platform of the VLT unit telescope \#1 (aka Antu) where it had first light on June 4/5, 2006. ${ }^{7}$

While the scientific potential of CRIRES could be demonstrated already in the first night, one more commissioning run was necessary to have the instrument sufficiently debugged and characterized so that it could be included into the call for proposals for ESO observing period P79, which started in April 2007. In parallel a

${ }^{*} 1.2 \lambda / D$ corresponds to $0.14 "$ at $\lambda \approx 4.6 \mu m(65.200 \mathrm{GHz})$, the location of the $C O$ fundamental band and the longest wavelength CRIRES is normally used.

${ }^{\dagger}$ The CRIRES science team consists of C. de Bergh, B. Gustafsson (chair), A. Hatzes, K. Hinkle and E. van Dishoek. 


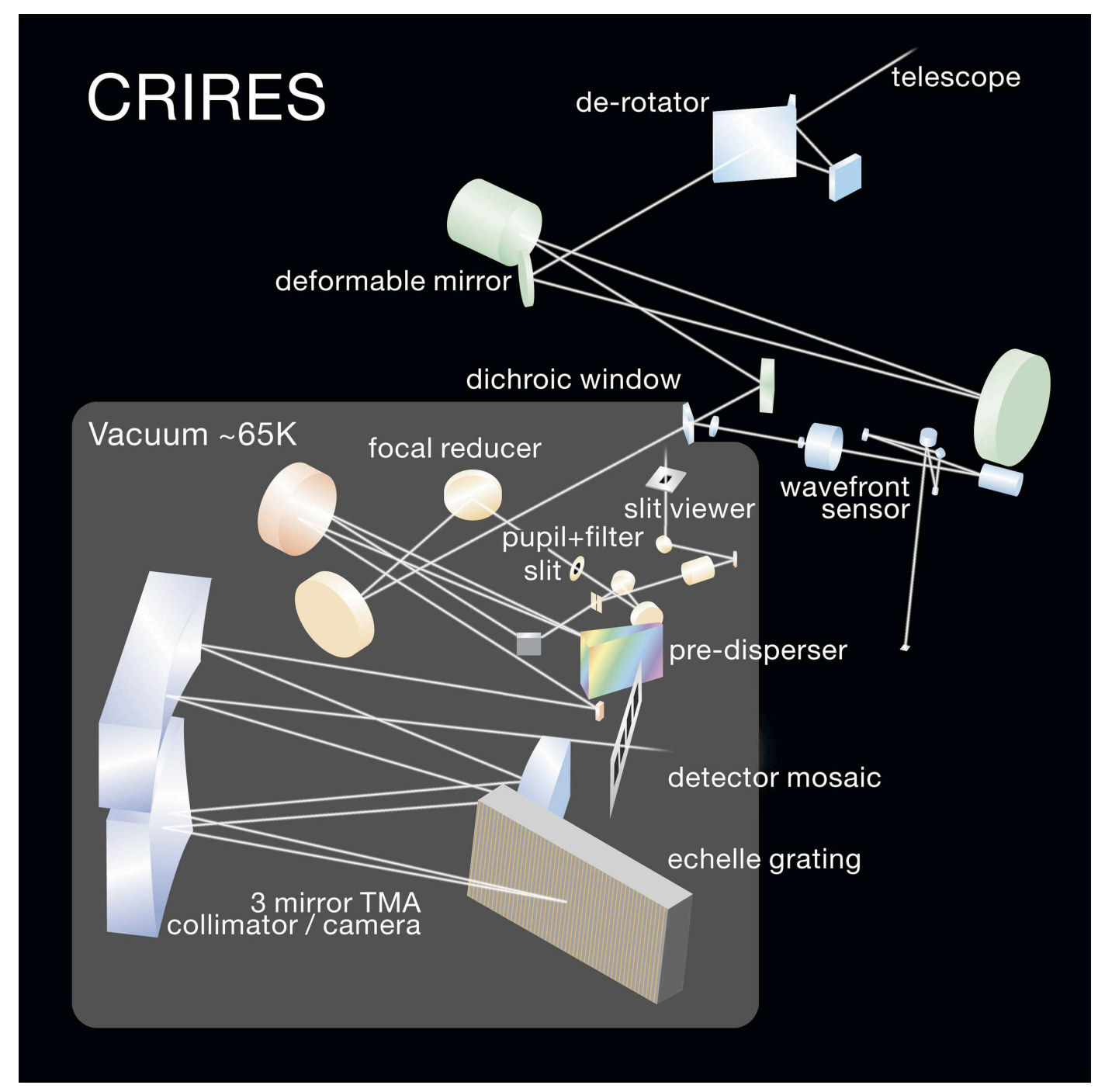

Figure 1. CRIRES optical design: The VLT Nasmyth focus (f\# 15) is close to the first mirror of the de-rotator assembly. A calibration unit (Neon arc-lamp, IR-HeNe-laser, Halogen lamp and an Infrared glower in combination with an integration sphere) serves for flat fielding and spectral calibration. In addition, gas-cells can be moved into the beam for calibration and for search for very small radial velocity changes similar to an iodine-cell in optical spectrographs. In future, in addition to the gas-cells retarders in a motorized mount can be placed to use CRIRES for spectro-polarimetry. The de-rotator is followed by the curvature sensing adaptive optics system with the deformable mirror on a kinematic gimbal mount. The entrance window to the cryostat is a dichroic, separating the visible light with high efficiency for the AO wavefront control. The entire cryogenic optical bench (highlighted by the grey background) is cooled with three Closed Cycle Coolers to $\approx 65 \mathrm{~K}$. The pre-slit optics of CRIRES consists of an all-reflective re-imager with a cold-pupil stop, reducing the f-ratio to $\mathrm{f} \#$ 7.5. Close to the cold pupil a Wollaston prism $\left(M g F_{2}\right)$ can be inserted, eventually with a linear polarizer compensating instrumental polarization. The slit-viewer (J,H \& $\mathrm{K}$ filters, $1 k x 1 k$ InSb Aladdin detector) gives a pixel scale of $0.05 \mathrm{arcsec} / \mathrm{pixel}$ and an un-vignetted field-of-view of $\approx 20 \mathrm{x} 40 \mathrm{arcsec}^{2}$. The main slit is continuously adjustable up to several arcsec with a closed loop encoder controlling the slit separation. The pre-disperser has a collimated beam diameter of $100 \mathrm{~mm}$ and uses a ZnSe prism in retro-reflection. The collimator mirror can be slightly tilted with a piezo. Order selection in the pre-dispersed spectrum is provided by a second intermediate slit located close to the small folding mirror. The main collimator, a three-mirror anastigmat (TMA), produces a $200 \mathrm{~mm}$ collimated beam which illuminates a R2 Echelle grating $(31.6 \mathrm{gr} / \mathrm{mm})$. The spectrum is imaged with a mosaic of four $1 k x 1 k$ InSb (Aladdin III) detectors. 
restricted call for proposals for early science verification (SV) was issued. SV-activities started as early as October 2006, but more activities in October and December (each split in commissioning and SV-activities) were necessary, before the "keys" of CRIRES could be handed over to the observatory staff. In April 2007 CRIRES greeted its first visiting astronomers and soon thereafter the first refereed paper based partially on CRIRES early SV-data appeared ${ }^{8}$ soon to be followed by a first letter, entirely based on CRIRES data. ${ }^{9}$

CRIRES cryogenic alignment was fairly easy with the all-metal-optics-approach. Focusing, however, required a series of openings of the cryostat. Internally there are four focal planes: the main slit, the slit-viewer detector, the intermediate slit and the science mosaic in the spectrograph focal plane. Finally, however, good focusing could be achieved. The slit-viewer has over its field $\leq 2.5$ pixel (FWHM) image quality. Monochromatic pointsources are imaged on the detector with typically 2 pixel FWHM. When illuminating the intermediate slit with the IR-HeNe-laser and closing it to the equivalent of a fraction of a pixel, then the laser line illuminates only one pixel in the focal plane, i.e. the spectrograph TMA plus grating are close to diffraction limited performance.

At this point CRIRES works to its nominal specifications, but a variety of technical issues deserve still to be addressed. Already now, however, it is clear that CRIRES is so popular that an upgrade plan has to be drafted in the near future, especially to take advantage of the development in the area of IR-array detectors.

\subsection{Some Lessons Learned}

CRIRES operations is quite complex in the sense that three control-loops have to be interfaced: the telescope tracking, the AO-guiding and the application of differential chromatic and other corrections to the tracking. It took more time than anticipated before all issues could be resolved. It should be noted, that the tracking on the slit viewer-image cannot be based on a normal evaluation of the photo-center of the program star, but comes from an educated guess derived from the wings of the PSF, as seen with the slit viewer detector (see below for typically achieved performance).

The first calibration plan was largely based on the assumption to use the spectral lamps to establish a physical model and then use telluric lines to establish corrections. While a physical model could be established ${ }^{10}$ and integrated into the operations software, the approach was still of limited use as the line density of telluric lines generally was found not to be sufficient and the reproducibility of the cold functions not good enough (the latter is being worked on).

The well known non-linearity of the Aladdin III detectors - especially in those chips read-out parallel to the dispersion - in combination with the limited precision of the cryogenic functions, especially the intermediate slit movement, led to some flat-fielding problems. Those could be mitigated by fine-tuning using the Piezo in the predisperser collimator, albeit at the expense of overhead times. Still high $\mathrm{S} / \mathrm{N}$ ratio stellar spectra $(S / N \geq 3-500)$ are possible ${ }^{8}$ (c.f. figure 2).

The requirements and their impact on the sub-system specifications for the intermediate slit had been underestimated. This could, however, be resolved by careful re-alignment and by adding an encoder to the intermediate slit movement.

Tests using fibers to inject light from the spectral calibration lamps at strategic positions in CRIRES have started. In the near future the fine positioning of grating and prism in CRIRES will be controlled by observing test spectra generated by these fibers (optical metrology).

\section{FREQUENCY CALIBRATION}

\subsection{The Perfect Calibration Lamp}

As mentioned above, calibration based entirely on telluric lines is not really an option. In table 2 the various possibilities are discussed. As the CRIRES spectral range spans more than two octaves, there is not one single method for calibration.

Table 2 gives a listing of calibrators and their relative merits. While the gas-cells in their spectral domain are close to perfect, the near-infrared calibration faces another problem: aiming at calibration precisions in line with a later deconvolution of spectra to fractions of a pixel requires a calibration source which truly uniformly illuminates the instrument pupil. The hollow-cathode lamps are way too faint to use integrating spheres. Direct 
Table 2. Frequency Calibration OPtions for High-Resolution Infrared Spectrographs

\begin{tabular}{|c|c|c|c|c|c|}
\hline source & \begin{tabular}{c}
\multicolumn{2}{c}{ range } \\
{$[\mathrm{GHz} / \mathrm{nm}]$}
\end{tabular} & brightness & line-density & $\begin{array}{l}\text { operational } \\
\text { constraints }\end{array}$ & $\begin{array}{l}\text { absolute } \\
\text { precision }\end{array}$ \\
\hline OH-airglow & $\begin{array}{c}150.000-315.000 \\
950-2000\end{array}$ & acceptable & marginal & $\begin{array}{l}\text { needs telescope } \\
\text { at night }\end{array}$ & $\begin{array}{l}\approx 20 \mathrm{~m} / \mathrm{s} \\
(\mathrm{tbc})\end{array}$ \\
\hline \multirow[t]{2}{*}{$\begin{array}{l}\text { atmospheric } \\
\text { absorption lines }\end{array}$} & $\begin{array}{c}158.000-315.000 \\
950-1900\end{array}$ & n.a. & $\begin{array}{l}\text { marginal to } \\
\text { acceptable }\end{array}$ & $\begin{array}{l}\text { not for long slit } \\
\text { mode }\end{array}$ & $\begin{array}{l}\approx 10 \mathrm{~m} / \mathrm{s} \\
(\mathrm{tbc})\end{array}$ \\
\hline & $\begin{array}{c}58,000-158.000 \\
1900-5200\end{array}$ & $\begin{array}{l}\text { acceptable } \\
\text { to good }\end{array}$ & $\begin{array}{l}\text { acceptable } \\
\text { to good }\end{array}$ & $\begin{array}{l}\text { needs telescope } \\
\text { at night }\end{array}$ & $\begin{array}{l}\approx 10 \mathrm{~m} / \mathrm{s} \\
(\mathrm{tbc})\end{array}$ \\
\hline $\begin{array}{l}\text { gas-cell } \\
\text { absorption }\end{array}$ & $\begin{array}{c}58.000-150.000 \\
2000-5200\end{array}$ & $\begin{array}{l}\text { acceptable } \\
\text { to good }\end{array}$ & $\begin{array}{l}\text { good to } \\
\text { very good }\end{array}$ & $\begin{array}{l}\text { cumbersome } \\
\text { operations }\end{array}$ & $\begin{array}{l}\leq 1 \mathrm{~m} / \mathrm{s} \\
\nu \text { absolute }\end{array}$ \\
\hline $\begin{array}{l}\text { gas discharge } \\
\text { lamps }\end{array}$ & $\begin{array}{c}120.000-315000 \\
950-2500\end{array}$ & very good & inappropriate & none & $\leq 10 \mathrm{~m} / \mathrm{s}$ \\
\hline $\begin{array}{l}\text { hollow cathode } \\
\text { lamps }\end{array}$ & $\begin{array}{c}120.000-315.000 \\
950-2500\end{array}$ & marginal & $\begin{array}{l}\text { good to } \\
\text { very good }\end{array}$ & $\begin{array}{l}\text { illumination } \\
\text { compromises }\end{array}$ & $\leq 10 \mathrm{~m} / \mathrm{s}$ \\
\hline
\end{tabular}

notes: For frequencies less than $158.000 \mathrm{GHz}$ the thermal emission from the core of the lines shines up in emission when observed against the (cold) night sky. For transitions having an optical depth $\tau \geq 1$ such lines can be very bright. This does not hold equally for the gas-cells as for the frequency range 150-315.000 GHz only overtone transitions exist which have $\tau \ll 1$. The gases to be used in CRIRES are $\mathrm{N}_{2} \mathrm{O}$ and $O C S$ which have a simple spectrum, measured by NIST against the Cesium time reference (see text).

CRIRES operations rely on ThAr hollow cathode lamps for $\nu \geq 120.000 \mathrm{GHz}(\lambda \leq 2500 \mathrm{~nm})$ and $\mathrm{N}_{2} \mathrm{O}$ for $\nu \leq 100.000 \mathrm{GHz}(\lambda \geq 3000 \mathrm{~nm})$. To that end, ESO took the initiative to establish a new line-catalog for the ThAr spectrum in the infrared. ${ }^{11}$

ilumation of the slit was tried, but only a small fraction can be illuminated. In June 2008 a major mechanical modification for the warm optics assembly was done, and now a fiber-optics system is used to illuminate 5-7 spots along the slit with the ThAr lamp. To account for the vastly different line intensities a motorized neutral density filter has been implemented.

CRIRES can hold up to two gas-cells (all metal sealed, $140 \mathrm{~mm}$ long $\mathrm{CaF}_{2}$-windows) which can be inserted into the beam by remote control. Gas-cells can be changed in daytime easily. A filling station to re-fill and check the internal pressure regularly has been built. The observatory has $\mathrm{CO}, \mathrm{N}_{2} \mathrm{O}, \mathrm{OCS}, \mathrm{NH}_{3}$ and $\mathrm{CH}_{4}$ available. Other gases are possible in ESO-cells or visitor gas-cells are possible in consultation with the observatory.

A final note on the precision of gas-cells. For all conceivable calibration applications of CRIRES rotational vibrational transitions of molecules in the vibrational ground-state are used. These gases behave very close to ideal gases. This implies that the density of molecules in the beam is invariable and relatively easy controllable. This situation is fundamentally different in the iodine gas-cells which are being used for radial velocity work. There iodine vapor is used in a heated cell and condensation on cooler parts (e.g. cell windows) can result in strong changes of the vapor pressure. Therefore, in case of infrared active gases, the transition frequencies are very close to those, which have been determined by NIST using heterodyne-techniques relative to the Cesium time standard. The spectra are relatively simple and lines which may consist of a blend can be positively excluded. The only conceivable process which may change transition frequencies in the gas cell in the case of an ideal gas is pressure shift. The pressure may change due to the external temperature. It has been estimated that this effect is less than $1 \mathrm{~m} / \mathrm{s}$. A more serious problem is pressure shift by air, in case the gas-cell has a leak. In the literature the is a good example. ${ }^{12}$ Figure $2 \mathrm{~b}$ in this paper shows the effect of pressure shift. A $\mathrm{PH}_{3}$-gas-cell line in the presence of a leak is measured relative to a gain-stabilized $\mathrm{CO}_{2}$-laser in a heterodyne setup. The kinks in the $\mathrm{PH}_{3}$-trace correspond to re-fillings of the cell. To control this problem, the pressure in the CRIRES gas-cells will be monitored as part of regular operations.

As CRIRES is now basically using spectral standards which have been referenced to the time standard, it appears logic, that for the future in high precision work the calibration should be in frequency $y^{\ddagger}$.

\footnotetext{
${ }^{\ddagger}$ Hertz is not only intellectually the clean unit for photon energy, it will also stop, once and forever the confusion
} 

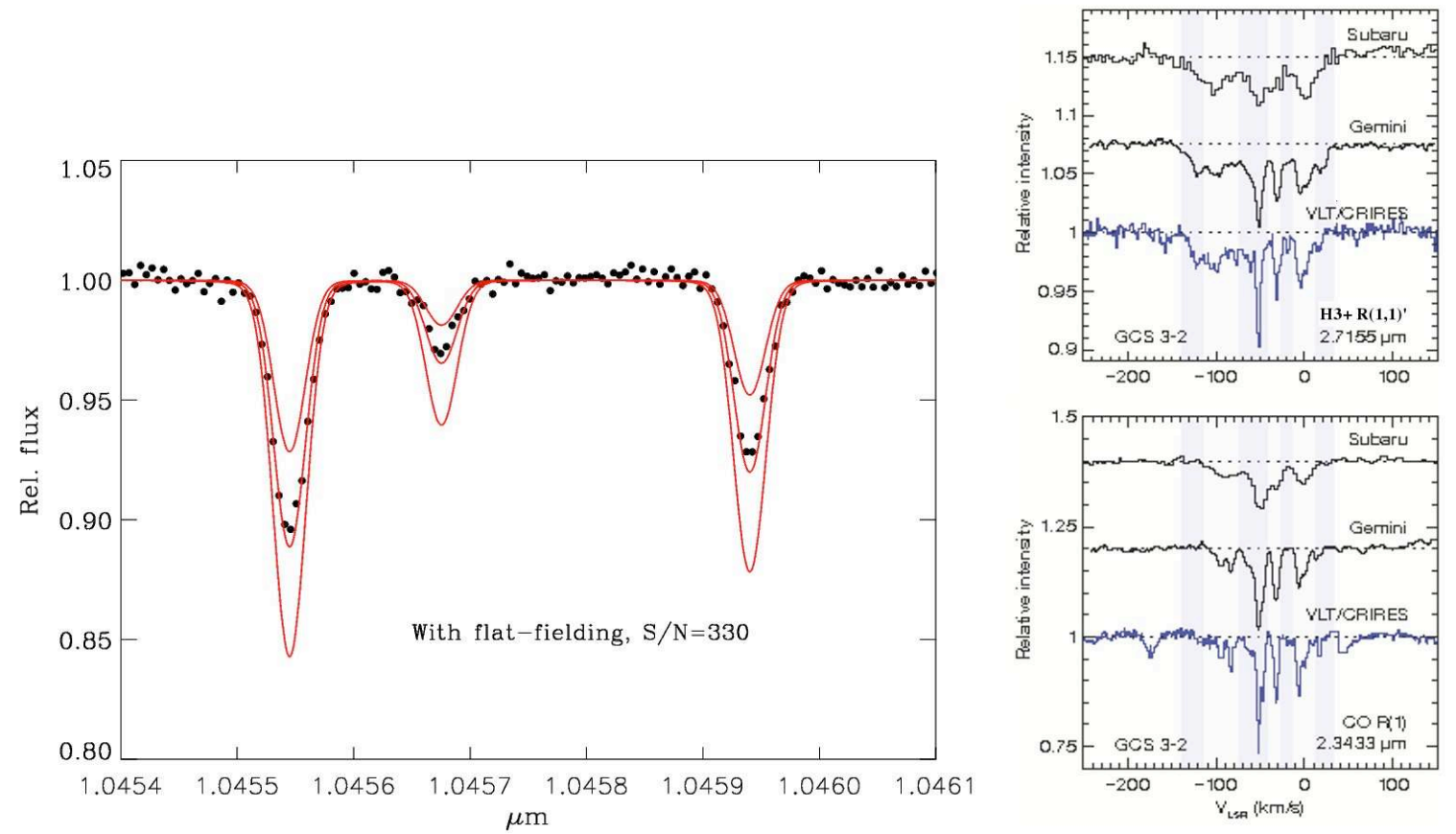

Figure 2. CRIRES, some results from early science verification The left part shows a spectrum of a metal poor star (i.e. clean continuum) with a sulfur triplet. The data and three models for different abundances are shown (P. Nissen, private communication). For the photospheric continuum a flatfield equivalent to a $\mathrm{S} / \mathrm{N}=330$ was achieved. The right part shows absorption spectra of an interstellar cloud taken against the galactic center source CGS 3-2 in the K-band (top: $H^{3+}$ and bottom: $C O$ ). Here the CRIRES data are shown in comparison to data taken with similar instrumentation at Subaru and Gemini having less spectral resolution (M. Goto, private communication). Most likely the lines are also not resolved with CRIRES.

\subsection{Absolute Stability}

The holy grail of extra-solar planet research is to find an Earth-like planet in an orbit corresponding to the habitable zone around a star in the solar neighborhood. At least for the moment with the radial-velocity technique, this is only feasible for M-dwarfs. Thus, high-resolution stable infrared spectrographs are in high demand. While specialized instrument concept studies are abundant, a practical test was done with CRIRES as to the stability and noise level which can be achieved. A 4.5 hour data set was taken during commissioning whereby the $\mathrm{SiO}$ overtone band at $75000 \mathrm{GHz}(4000 \mathrm{~nm})$ in a late type star is measured relative to an $\mathrm{N}_{2} \mathrm{O}$-band in our atmosphere serving as reference for the local standard of rest. After careful analysis using correlation techniques $^{13}$ it was found that the $1 \sigma$ error in radial velocity as measured with CRIRES through a 4.5 hour interval is $\approx 8 \mathrm{~m} / \mathrm{s}$ (this includes the uncertainty introduced in the telluric lines by Doppler-shift due to unknown local wind above the telescope).

Meanwhile, the internal stability of CRIRES has been improved, especially by adding mK temperature control to the grating. Also using dedicated gas-cells improvements are possible by providing more lines and by ensuring zero relative velocity between the gas with its line system and the LSR.

\section{EXAMPLES FROM SCIENCE VERIFICATION AND OPERATION}

A general overview including examples from the slit-viewing camera competing with AO-imagers is already available. ${ }^{14}$ Picked from by now a cornucopia of results figure 2 shows the very first publishable result and a

between vacuum and air wavelengths which plagues all catalog and all modeling efforts based on line lists referring to wavelength. 
comparison of CRIRES (or better its spectral resolution) to other IR-spectrographs when looking at an interstellar cold cloud in absorption.

Particularly interesting are the results published from the first visiting observer programme ${ }^{15}$ who have observed the $C O$-fundamental band at $65.100 \mathrm{GHz}$ in emission from suspected proto-planetary discs in a sample of four stars. By carefully analyzing the cross-dispersion profile and doing an analysis of the photo-center as a function of frequency they find spatial information down to the milli-arcsec level, or typically 0.1 AU in the respective objects. Observing with variable position angle of the slit yields extremely high confidence, that these structures are not an artifact but real.

For CRIRES there is by now a fully operational pipeline, which has also been released for general public use. FOr operations and the preparation of observing proposals the most important tool is the exposure time calculator. For its core functionality it includes a realistic model of the AO-performance. It also calculates precisely the interfering telluric absorption spectrum and applies the precise Dopplershift to be expected fo the date of observations. Over the year the velocity can change up to $\pm 30 \mathrm{~km} / \mathrm{s}$ and the right choice of observing date can contribute substantially to the success of an observing program.

\section{CONCLUSIONS AND OUTLOOK}

CRIRES, the VLT infrared high resolution Echelle spectrograph has been put in operation at the observatory. The instrument has already produced science results in various fields from abundances in the galactic center to the gas coma in local comets. Frequency stability and calibration down to a level of $\approx 8-10 \mathrm{~m} / \mathrm{s}$ have been demonstrated, already shortly after first light. CRIRES is still plagued by some technical problems, so that its full potential is not yet unleashed. Various ideas for upgrades are considered and an upgrade plan will soon be drafted.

Of particular interest is the implementation of the spectro-polarimetric mode ${ }^{16}$ which is targeted for end of 2008. This will include also a calibration unit to allow to establish a physical model for the instrumental polaristaion.

CRIRES has demonstrated, that similar instruments for the next generation of large telescopes could be highly valuable. For the E-ELT the spectroscopic arm of the METIS ${ }^{17}$ instrument will cover frequencies less than $300.000 \mathrm{GHz}$, i.e. most of the fundamental rotational-vibrational transitions observable from the ground. Extrapolating results ${ }^{15}$ from CRIRES the diffraction limit of an E-ELT will allow to resolve spatial structures down to few $100 \mu$ arcsec.

\section{ACKNOWLEDGMENTS}

This project has benefit a lot from support of the dedicated staff of the La Silla-Paranal observatory, and many other colleagues in the community especially those contributing to the HITRAN database. Particularly the authors gratefully acknowledge the following online resources for access to spectroscopic data bases:

the US - National Institute of Standards and Technology (NIST):

http://physics.nist.gov/PhysRefData/wavenum/html/spect.html

the online access to the archive of Solar Fourier Transform Spectrometer data from the Kitt-Peak Solar telescope through ftp://ftp.noao.edu/fts. The NSO/Kitt Peak FTS data being used in the CRIRES project were produced by NSF/NOAO.

The atlas of atomic lines maintained by Peter van Hoof, Physics Department Queen's University Belfast, Northern Ireland accessible through: http://www.pa.uky.edu/ peter/atomic/

\section{REFERENCES}

[1] Käufl, H.U., Ballester, P., Biereichel, P., et al. (2004): SPIE 5492, pp. 1218-1227

[2] Käufl, H.U., Siebenmorgen, R., Moorwood, A. (2005): High Resolution Infrared Spectroscopy in Astronomy, Proceedings of an ESO Workshop held at Garching, Germany, 18-21 November 2003 Springer Berlin / Heidelberg

[3] Paufique, J., Biereichel, P., Donaldson, R., et al. (2004): SPIE 5490, 216 
[4] Arsenault, R., Alonso, J., Bonnet, H., et al (2003): SPIE 4839, 174

[5] Paufique, J., Biereichel, P., Donaldson, R., et al. (2006): SPIE 6272, 36

[6] Dorn, R. J., Finger, G., Huster, G./ et al. (2004): SPIE 5499, 510

[7] Käufl, H.U., Amico, P., Ballester, P., et al. (2006): The Messenger, 126, 32

[8] Nissen, P. E., Akerman, C., Asplund, M. et al. (2007): A\&A 469, 319

[9] Reiners, A., Seifahrt, A., Käufl, H.U. et al. (2007): A\&A 471, L5

[10] Bristow, P., Kerber, F., Rosa, M. R., et al. (2006): SPIE 6270, 58B

[11] Kerber, F., Nave, G., \& Sansonetti, C.J. (2008): ApJS, in press

[12] Glenar, D. A., Käufl, H. U., Deming, D., Kostiuk, T., \& Mumma, M. J. (1988): Adv. Helio- and Asteroseismology: I.A.U.Symp. 123,481

[13] Seifahrt, A., \& Käufl, H. U. (2008): High Precision Radial Velocity Measurements in the NIR A\&A, submitted

[14] Siebenmorgen, K., Smette, A., Käufl, H.U. et al. (2007): The Messenger 12817

[15] Pontoppidan, K., Blake, G.A., van Dishoeck, E.F. et al. (2008): ApJ in press or arXiv:0805.3314

[16] Kaeufl, H.U., Moorwood, A.F.M. \& Pirard, J.F. (2003): SPIE 4843, 223

[17] Brandl, B., et al. (2008): This proceedings 